Pacific

Journal of

Mathematics

BOSONIC REALIZATIONS OF HIGHER-LEVEL TOROIDAL LIE ALGEBRAS

NAIHUAN Jing, KaILASh MisRa AND ShaObin TAN

Volume $219 \quad$ No. 2

April 2005 


\title{
BOSONIC REALIZATIONS OF HIGHER-LEVEL TOROIDAL LIE ALGEBRAS
}

\author{
Naihuan Jing, Kailash MisRa And ShaObin TAN
}

\begin{abstract}
We construct realizations for the 2-toroidal Lie algebra associated with the Lie algebra $A_{1}$ using vertex operators based on bosonic fields. In particular our construction realizes higher-level representations of the 2-toroidal algebra for any given pair of levels $\left(k_{0}, k_{1}\right)$ with $k_{0} \neq 0$. We also construct a smaller module of level $\left(k_{0}, 0\right)$ for the toroidal algebra from the Fock space using certain screening vertex operator, and this later representation generalizes the higher-level construction of the affine Lie algebra $\widehat{\mathfrak{s l}}_{2}$.
\end{abstract}

\section{Introduction}

Toroidal Lie algebras are a natural generalization of the affine Kac-Moody algebras introduced by Moody, Rao and Yokonuma [Moody et al. 1990]. Let $A=$ $\mathbb{C}\left[s, s^{-1}, t, t^{-1}\right]$ be the ring of Laurent polynomials in commuting variables. By definition a 2-toroidal Lie algebra is a perfect central extension of the iterated loop algebra $\mathfrak{g} \otimes A$, where $\mathfrak{g}$ is a finite-dimensional simple Lie algebra over $\mathbb{C}$.

Let $\Omega_{A} / d A$ be the Kähler differentials of $A$ modulo the exact forms. The universal central extension of the iterated loop algebra is given by

$$
T(\mathfrak{g})=(\mathfrak{g} \otimes A) \oplus \Omega_{A} / d A .
$$

Any 2-toroidal Lie algebra is a homomorphic image of this toroidal Lie algebra. The center of $T(\mathfrak{g})$ is $\Omega_{A} / d A$, which is a infinite-dimensional vector space. The Laurent polynomial ring $A$ induces a natural $\mathbb{Z}^{2}$-gradation on $T(\mathfrak{g})$. For the center we have $\Omega_{A} / d A=\bigoplus_{\sigma \in \mathbb{Z}^{2}} \mathscr{E}(\mathfrak{g})_{\sigma}$, with $\operatorname{dim} \mathscr{E}_{\sigma}=1$ if $\sigma \neq(0,0)$ and 2 if $\sigma=(0,0)$. We denote by $c_{0}$ and $c_{1}$ the two standard degree-zero central elements in the toroidal Lie algebra $T(\mathfrak{g})$. A module of $T(\mathfrak{g})$ is called a level- $\left(k_{0}, k_{1}\right)$ module if the standard center $\left(c_{0}, c_{1}\right)$ acts as $\left(k_{0}, k_{1}\right)$ for some complex numbers $k_{0}$ and $k_{1}$. Here we study the level- $\left(k_{0}, k_{1}\right)$ modules for $k_{0} \neq 0$.

MSC2000: 17B65, 17B69,

Keywords: toroidal Lie algebra, vertex operator, bosonic realization.

Jing acknowledges the partial support from the NSF and NSA. Misra acknowledges the partial support from the NSA. Tan was supported by the National Science Foundation of China, No. 10371100. 
In various constructions of the affine Lie algebra $\widehat{\mathfrak{s l}}_{2}$ the free field representation is of particular use in its applications. Wakimoto [1986] and Feigen and Frenkel [1988] first gave a general construction for the general case, and later Nemenschansky [1989] gave an invariant form in the special case. Though the two forms can be interchanged by a nontrivial map, we realized that the later form is better for our purpose in the toroidal cases. The operators in question have the form $e^{A}(B+C)$, where $A, B, C$ are generating functions of the scaled Heisenberg operators. One of the nice things is that all root generators in the toroidal algebra associated with the Lie algebra $\mathfrak{s l}_{2}$ can be represented by this type of vertex operators. In our construction we have fully used this simplicity and make all calculations in a uniform manner.

As we mentioned earlier, toroidal algebras are generalizations of finite-dimensional Lie algebras, like affine Lie algebras. This similarity is constantly kept in mind as we study their structure and representation theory. Some other basic references related to our work include [Berman and Billig 1999; Eswara Rao and Moody 1994; Fabbri and Moody 1994; Larsson 1999; Moody et al. 1990; Tan 1999]. Our aim in this paper is to give a higher-level representation for the simplest nontrivial example: the 2-toroidal Lie algebra. Our construction generalizes previous work on higher-level representations of the affine Lie algebra $\widehat{\mathfrak{s l}}_{2}$.

In Section 2 we define the toroidal Lie algebra and state the MRY-presentation [Moody et al. 1990] of the toroidal algebra in terms of generators and relations. The algebra structure is expressed in terms of formal power series identities. We also state some results in this section to be used later. In Section 3 we start with a finite-rank lattice with a symmetric bilinear form and define a Fock space and some vertex operators, which in turn give representations of the toroidal Lie algebra of type $A_{1}$, and also a level- $\left(k_{0}, 0\right)$ module with $k_{0} \neq 0$ for the double affine algebra of type $A_{1}$. In Section 4 we study the structure of the Fock space for the toroidal Lie algebra by using certain screening vertex operators, thus generalizing the higherlevel representation of the affine algebra $\widehat{\mathfrak{s l}}_{2}$ to the toroidal Lie algebra.

\section{Toroidal Lie algebras}

Let $\mathfrak{s l}_{2}$ be the 3-dimensional simple Lie algebra over the complex numbers and

$$
A=\mathbb{C}\left[s, s^{-1}, t, t^{-1}\right]
$$

the ring of Laurent polynomials in commuting variables. We consider the iterated loop algebra

$$
\mathfrak{g}=\mathfrak{s l}_{2} \otimes A .
$$

A toroidal Lie algebra of type $A_{1}$ is a perfect central extension of the iterated loop algebra $\mathfrak{g}$, which is often an infinite-dimensional central extension. Let $\Omega_{A}$ be the 
$A$-module of differentials with differential mapping $d: A \rightarrow \Omega_{A}$, such that

$$
d\left(f_{1} f_{2}\right)=\left(d f_{1}\right) f_{2}+f_{1}\left(d f_{2}\right) \text { for all } f_{1}, f_{2} \text { in } A .
$$

Let ${ }^{-}: \Omega_{A} \rightarrow \Omega_{A} / d A$ be the canonical linear map for which $\overline{d f}=0$ for all $f \in A$. Endow the vector space

$$
T\left(A_{1}\right):=\left(\mathfrak{s l}_{2} \otimes A\right) \oplus \Omega_{A} / d A
$$

with the bracket operation defined by

$$
\left[x \otimes f_{1}, y \otimes f_{2}\right]=[x, y] \otimes f_{1} f_{2}+(x, y) \overline{f_{2} d f_{1}},
$$

for $x, y \in \mathfrak{s l}_{2}, f_{1}, f_{2} \in A$, where $(\cdot, \cdot)$ is the trace form and $\Omega_{A} / d A$ is central. From [Moody et al. 1990] we know that $T\left(A_{1}\right)$ is a perfect Lie algebra and is the universal central extension of the iterated loop algebra $\mathfrak{s l}_{2} \otimes A$. Therefore any toroidal Lie algebra of type $A_{1}$ is a homomorphic image of $T\left(A_{1}\right)$. The gradation of the polynomial ring $A$ gives a natural $\mathbb{Z}^{2}$-gradation to the toroidal Lie algebra

$$
T\left(A_{1}\right):=\bigoplus_{\sigma \in \mathbb{Z}^{2}} T\left(A_{1}\right)_{\sigma}
$$

where $T\left(A_{1}\right)_{\sigma}$ is spanned by $x \otimes s^{m_{0}} t^{m_{1}}, \overline{s^{m_{0}} t^{m_{1}} s^{-1} d s}$ and $\overline{s^{m_{0}} t^{m_{1}} t^{-1} d t}$ for $\sigma=$ $\left(m_{0}, m_{1}\right) \in \mathbb{Z}^{2}$ and $x \in \mathfrak{s l}_{2}$. The condition $\overline{d f}=0$ for all $f \in A$ implies that $m_{0} \overline{s^{m_{0}} t^{m_{1}} s^{-1} d s}+m_{1} \overline{s^{m_{0}} t^{m_{1}} t^{-1} d t}=0$ for all $m_{0}, m_{1} \in \mathbb{Z}$. Therefore the dimension of $T\left(A_{1}\right)_{\sigma}$ is 4 if $\sigma \neq(0,0)$ and 5 if $\sigma=(0,0)$. In particular, $T\left(A_{1}\right)_{(0,0)}$ is spanned by $x \otimes 1$ for $x \in \mathfrak{s l}_{2}$, and central elements $\overline{s^{-1} d s}, \overline{t^{-1} d t}$. We denote these two degree-zero central elements by $c_{0}$ and $c_{1}$.

The most interesting quotient algebra of the toroidal Lie algebra $T\left(A_{1}\right)$ is the double affine algebra denoted by $T_{0}\left(A_{1}\right)$, that is, the toroidal Lie algebra of type $A_{1}$ with a two-dimensional center. The double affine algebra is the quotient of $T\left(A_{1}\right)$ modulo all the central elements with degree other than zero. In fact, $T_{0}\left(A_{1}\right)$ has the realization

$$
T_{0}\left(A_{1}\right)=\left(\mathfrak{s l}_{2} \otimes A\right) \oplus \mathbb{C} c_{0} \oplus \mathbb{C} c_{1}
$$

with the Lie product

$$
\left[x \otimes f_{1}, y \otimes f_{2}\right]=[x, y] \otimes f_{1} f_{2}+\Phi\left(f_{2} \partial_{s} f_{1}\right) c_{0}+\Phi\left(f_{2} \partial_{t} f_{1}\right) c_{1}
$$

for all $x, y \in \mathfrak{s l}_{2}$ and $f_{1}, f_{2} \in A$, where $\Phi$ is the linear functional on $A$ defined by

$$
\Phi\left(s^{k} t^{m}\right)= \begin{cases}0, & \text { if }(k, m) \neq(0,0) \\ 1, & \text { if }(k, m)=(0,0)\end{cases}
$$

for all $k, m \in \mathbb{Z}$. 
Definition 2.1. If $M$ is a module for a toroidal Lie algebra of type $A_{1}$, we call $M$ a level- $\left(k_{0}, k_{1}\right)$ module for some complex numbers $k_{0}, k_{1}$ if the degree-zero central elements $c_{0}, c_{1}$ act on $M$ as constants $k_{0}, k_{1}$.

In this paper we give a concrete construction for a level- $\left(k_{0}, k_{1}\right)$ module with $k_{0} \neq 0$ for the toroidal Lie algebra $T\left(A_{1}\right)$ and for the double affine algebra $T_{0}\left(A_{1}\right)$.

Let $\left\{x_{ \pm}, h\right\}$ be the standard basis of $\mathfrak{s l}_{2}$. Also let $\left(a_{i j}\right)_{2 \times 2}$ be the generalized Cartan matrix of the affine algebra $A_{1}^{(1)}$ and

$$
Q:=\mathbb{Z} \alpha_{0}+\mathbb{Z} \alpha_{1}
$$

its root lattice. The toroidal Lie algebra $T\left(A_{1}\right)$ has a presentation [Moody et al. 1990] with generators $\phi, \alpha_{i}(k)$ and $x_{k}\left( \pm \alpha_{i}\right)$, for $k \in \mathbb{Z}$ and $i=0,1$, and the following relations, for $k, m \in \mathbb{Z}$ and $i, j=0,1$ :

(R0) $\left[\phi, \alpha_{i}(k)\right]=0=\left[\phi, x_{k}\left( \pm \alpha_{i}\right)\right]$;

(R1) $\left[\alpha_{i}(k), \alpha_{j}(m)\right]=k a_{i j} \delta_{k+m, 0} \phi$;

(R2) $\left[\alpha_{i}(k), x_{m}\left( \pm \alpha_{j}\right)\right]= \pm a_{i j} x_{k+m}\left( \pm \alpha_{j}\right)$;

(R3) $\left[x_{k}\left(\alpha_{i}\right), x_{m}\left(-\alpha_{j}\right)\right]=-\delta_{i j}\left\{\alpha_{i}(k+m)+k \delta_{k+m, 0} \phi\right\}$;

(R4) $\left[x_{k}\left(\alpha_{i}\right), x_{m}\left(\alpha_{i}\right)\right]=0=\left[x_{k}\left(-\alpha_{i}\right), x_{m}\left(-\alpha_{i}\right)\right]$;

$$
\left(\operatorname{ad} x_{0}\left(\alpha_{i}\right)\right)^{3} x_{m}\left(\alpha_{j}\right)=0 \text { if } i \neq j ; \quad\left(\operatorname{ad} x_{0}\left(-\alpha_{i}\right)\right)^{3} x_{m}\left(-\alpha_{j}\right)=0 \text { if } i \neq j .
$$

The Lie algebra isomorphism $\psi$ between the two presentations of $T\left(A_{1}\right)$ is given by

$$
\begin{aligned}
\not & \mapsto \overline{s^{-1} d s}, \\
x_{m}\left( \pm \alpha_{1}\right) & \mapsto \pm x_{ \pm} \otimes s^{m}, \\
x_{m}\left( \pm \alpha_{0}\right) & \mapsto \pm x_{\mp} \otimes s^{m} t^{ \pm 1}, \\
\alpha_{1}(k) & \mapsto h \otimes s^{k}, \\
\alpha_{0}(k) & \mapsto-h \otimes s^{k}+\overline{s^{k} t^{-1} d t} .
\end{aligned}
$$

Therefore, the degree-zero central elements are $c_{0}=\phi$ and $c_{1}=\delta(0)$, where $\delta=\alpha_{0}+\alpha_{1}$ is the null root in $Q$. We will identify the two presentations of the toroidal Lie algebra $T\left(A_{1}\right)$ via this isomorphism $\psi$.

Following [Moody et al. 1990], we introduce a $\mathbb{Z} \times Q$-gradation on $T\left(A_{1}\right)$ by assigning $\operatorname{deg} \phi=(0,0), \operatorname{deg} \alpha_{i}(k)=(k, 0), \operatorname{deg} x_{k}\left( \pm \alpha_{i}\right)=\left(k, \pm \alpha_{i}\right)$, with $i=0,1$ and $k \in \mathbb{Z}$. We denote by $T_{k}^{\alpha}$ the subspace of $T\left(A_{1}\right)$ spanned by the elements with degree $(k, \alpha)$ for $k \in \mathbb{Z}, \alpha \in Q$. Then, under the isomorphism $\psi$, we have $\psi^{-1}\left(\overline{s^{k} t^{-1} d t}\right)=\delta(k) \in T_{k}^{0}$ and $\psi^{-1}\left(\overline{s^{k} t^{r} s^{-1} d s}\right) \in T_{k}^{r \delta}$. 
Let $z, w, z_{1}, z_{2}, \ldots$ be formal variables. We define formal power series with coefficients from the toroidal Lie algebra $T\left(A_{1}\right)$ :

$$
\begin{aligned}
\alpha_{i}(z) & =\sum_{n \in \mathbb{Z}} \alpha_{i}(n) z^{-n-1}, \\
x\left( \pm \alpha_{i}, z\right) & =\sum_{n \in \mathbb{Z}} x_{n}\left( \pm \alpha_{i}\right) z^{-n-1},
\end{aligned}
$$

for $i=0,1$. Then the Lie algebra structure of $T\left(A_{1}\right)$ can be expressed in terms of the following power series identities:

$\left(\mathrm{R}^{\prime}\right)\left[\phi, \alpha_{i}(z)\right]=0=\left[\phi, x\left( \pm \alpha_{i}, z\right)\right]$;

$\left(\mathrm{R} 1^{\prime}\right)\left[\alpha_{i}(z), \alpha_{j}(w)\right]=a_{i j} z^{-1} \partial_{w} \delta\left(\frac{w}{z}\right) \phi ;$

$\left(\mathrm{R} 2^{\prime}\right)\left[\alpha_{i}(z), x\left( \pm \alpha_{j}, w\right)\right]= \pm a_{i j} x\left( \pm \alpha_{j}, w\right) z^{-1} \delta\left(\frac{w}{z}\right)$;

$\left(\mathrm{R}^{\prime}\right)\left[x\left(\alpha_{i}, z\right), x\left(-\alpha_{j}, w\right)\right]=-\delta_{i j}\left\{\alpha_{i}(w) z^{-1} \delta\left(\frac{w}{z}\right)+z^{-1} \partial_{w} \delta\left(\frac{w}{z}\right) \phi\right\}$;

$\left(\mathrm{R} 4^{\prime}\right)\left[x\left(\alpha_{i}, z\right), x\left(\alpha_{i}, w\right)\right]=0=\left[x\left(-\alpha_{i}, z\right), x\left(-\alpha_{i}, w\right)\right]$;

$\left(\operatorname{ad} x\left(\alpha_{i}, z_{1}\right)\right)\left(\operatorname{ad} x\left(\alpha_{i}, z_{2}\right)\right)\left(\operatorname{ad} x\left(\alpha_{i}, z_{3}\right)\right) x\left(\alpha_{j}, z_{4}\right)=0 \quad$ if $i \neq j ;$

$\left(\operatorname{ad} x\left(-\alpha_{i}, z_{1}\right)\right)\left(\operatorname{ad} x\left(-\alpha_{i}, z_{2}\right)\right)\left(\operatorname{ad} x\left(-\alpha_{i}, z_{3}\right)\right) x\left(-\alpha_{j}, z_{4}\right)=0 \quad$ if $i \neq j$.

Finally, we recall a result from [Moody et al. 1990] that will be used in the next section.

Proposition 2.2. Suppose $\mathscr{L}$ is a Lie algebra over $\mathbb{C}$ graded by $\mathbb{Z} \otimes Q$, and $\phi$ : $T\left(A_{1}\right) \rightarrow \mathscr{L}$ is a surjective graded homomorphism of Lie algebras such that

(i) $\phi$ is injective on $T_{n}^{\alpha}$ for all $n \in \mathbb{Z}$ and real root $\alpha$,

(ii) $\phi(\delta(k)) \neq 0$ for all $k$ and $\left.\phi\right|_{\mathbb{C} \delta(0)+\mathbb{C} \phi}$ is injective, and

(iii) for all nonzero integers $k, m$,

$$
\begin{array}{r}
\phi\left(\left[x_{m}\left(\alpha_{1}+k \delta\right), x_{0}\left(-\alpha_{1}\right)\right]-\left[x_{0}\left(\alpha_{1}+k \delta\right), x_{m}\left(-\alpha_{1}\right)\right]\right) \neq 0, \\
\phi\left(\left[x_{1}\left(\alpha_{1}+k \delta\right), x_{-1}\left(-\alpha_{1}\right)\right]-\left[x_{-1}\left(\alpha_{1}+k \delta\right), x_{1}\left(-\alpha_{1}\right)\right]\right) \neq 0 .
\end{array}
$$

Then $\phi$ is an isomorphism, where $x_{m}\left( \pm \alpha_{1}+k \delta\right):=\psi^{-1}\left( \pm x_{ \pm} \otimes s^{m} t^{k}\right)$.

Proposition 2.3. Suppose $\mathscr{L}$ is a Lie algebra over $\mathbb{C}$ graded by $\mathbb{Z} \otimes Q$, and $\phi$ : $T\left(A_{1}\right) \rightarrow \mathscr{L}$ is a surjective graded homomorphism of Lie algebras such that

(i) $\phi$ is injective on $T_{n}^{\alpha}$ for all $n \in \mathbb{Z}$ and real root $\alpha$,

(ii) $\phi(\delta(k))=0$ for all $k \neq 0$ and $\left.\phi\right|_{\mathbb{C} \delta(0)+\mathbb{C} \phi}$ is injective, and

(iii) for all nonzero integers $k, m$,

$$
\begin{aligned}
\phi\left(\left[x_{m}\left(\alpha_{1}+k \delta\right), x_{0}\left(-\alpha_{1}\right)\right]-\left[x_{0}\left(\alpha_{1}+k \delta\right), x_{m}\left(-\alpha_{1}\right)\right]\right) & =0, \\
\phi\left(\left[x_{1}\left(\alpha_{1}+k \delta\right), x_{-1}\left(-\alpha_{1}\right)\right]-\left[x_{-1}\left(\alpha_{1}+k \delta\right), x_{1}\left(-\alpha_{1}\right)\right]\right) & =0,
\end{aligned}
$$


Then $\mathscr{L}$ is isomorphic to the double affine algebra $T_{0}\left(A_{1}\right)$.

Proof. We only need to show that the set of nonzero-degree central elements of the toroidal Lie algebra $T\left(A_{1}\right)$ is in the kernel of $\phi$. Indeed, under the isomorphism $\psi$ of the toroidal Lie algebras, we see that $\delta(k)=\psi^{-1}\left(\overline{s^{k} t^{-1} d t}\right)$ and

$$
\begin{aligned}
{\left[x_{m}\left(\alpha_{1}+k \delta\right), x_{0}\left(-\alpha_{1}\right)\right]-\left[x_{0}\left(\alpha_{1}+k \delta\right), x_{m}\left(-\alpha_{1}\right)\right] } & =-m \psi^{-1}\left(\overline{s^{m} t^{k} s^{-1} d s}\right), \\
{\left[x_{1}\left(\alpha_{1}+k \delta\right), x_{-1}\left(-\alpha_{1}\right)\right]-\left[x_{-1}\left(\alpha_{1}+k \delta\right), x_{1}\left(-\alpha_{1}\right)\right] } & =-2 \psi^{-1}\left(\overline{t^{k} s^{-1} d s}\right),
\end{aligned}
$$

but, from [Moody et al. 1990], the elements $\overline{s^{p} t^{q} s^{-1} d s}, \overline{s^{p} t^{-1} d t}$ and $\overline{s^{-1} d s}$ for $(p, q) \in \mathbb{Z} \times(\mathbb{Z} \backslash\{0\})$ form a basis of the center for the toroidal Lie algebra $T\left(A_{1}\right)$. The assumption implies that the nonzero-degree central elements $\psi^{-1}\left(\overline{s^{p} t^{q} s^{-1} d s}\right)$ and $\psi^{-1}\left(\overline{s^{q} t^{-1} d t}\right)$ are in the kernel of the homomorphism $\phi$ for

$$
(p, q) \in \mathbb{Z} \times(\mathbb{Z} \backslash\{0\}) .
$$

\section{Representations of the toroidal algebra}

In this section we give two bosonic realizations for the toroidal Lie algebra $T\left(A_{1}\right)$. Let $k_{0}$ be a fixed complex number with $k_{0} \neq 0$, and $\Gamma$ a finite rank lattice with a symmetric $\mathbb{C}$-valued $\mathbb{Z}$-bilinear form $(\cdot, \cdot)$. We extend the form to a $\mathbb{C}$-bilinear form on the vector space $H=\mathbb{C} \otimes_{\mathbb{Z}} \Gamma$. Let $\Gamma_{0}$ be a fixed integral sublattice of $\Gamma$. We define

$$
\Gamma_{0}^{\star}=\left\{\alpha \in H ;\left(\alpha, \Gamma_{0}\right) \subset \mathbb{Z}\right\}
$$

Then $\Gamma_{0} \subset \Gamma_{0}^{\star}$. Let

$$
\mathscr{H}=\langle h(n), \phi \mid h \in H, n \in \mathbb{Z}\rangle,
$$

with $H=\mathbb{C} \otimes_{\mathbb{Z}} \Gamma$, be the affinization of the vector space $H$, defined with the Lie product

$$
[\alpha(m), \beta(n)]=m(\alpha, \beta) \delta_{m+n, 0} \phi
$$

for $m, n \in \mathbb{Z}, \alpha, \beta \in \Gamma$, and $\not$ central. We define the Fock space

$$
V:=\mathbb{C}\left[\Gamma_{0}^{\star}\right] \otimes S\left(\mathscr{H}^{-}\right),
$$

where $S\left(\mathscr{H}^{-}\right)$is the symmetric algebra on $\mathscr{H}^{-}:=\langle h(n) \mid n<0\rangle$, and

$$
\mathbb{C}\left[\Gamma_{0}^{\star}\right]=\bigoplus_{\alpha \in \Gamma_{0}^{\star}} \mathbb{C} e^{\alpha}
$$

is the group algebra on the additive subgroup $\Gamma_{0}^{\star}$ of the vector space $H$. Then $V$ has a natural module structure for the Lie algebra $\mathscr{H}$ and the group algebra $\mathbb{C}\left[\Gamma_{0}^{\star}\right]$ with the actions defined by making $\phi$ act as $k_{0}, h(-n)$ act as multiplication, and $h(n)$ act as a partial differential operator, for $n>0, h \in H$, so that

$$
[\alpha(m), \beta(n)]=m k_{0}(\alpha, \beta) \delta_{m+n, 0}
$$


for all $\alpha, \beta \in H$ and $m, n \in \mathbb{Z}$. Moreover $\alpha(0)$ acts as a partial differential operator on $\mathbb{C}\left[\Gamma_{0}^{\star}\right]$ for which $\left[\alpha(0), e^{\beta}\right]=(\alpha, \beta) e^{\beta}$. Therefore $\alpha(0) . \beta=(\alpha, \beta)$ for $\alpha, \beta \in H$.

With a formal variable $z$, and $\alpha, \beta \in H$, we define fields

$$
\begin{aligned}
\alpha(z) & =\sum_{n \in \mathbb{Z}} \alpha(n) z^{-n-1}, \\
\alpha(z)_{+} & =\sum_{n<0} \alpha(n) z^{-n-1}, \\
\overline{\beta(z)} & =\beta+\beta(0) \log z-\sum_{n \neq 0} \frac{\beta(n)}{n} z^{-n}, \\
\overline{\beta(z)}_{+} & =\beta-\sum_{n<0} \frac{\beta(n)}{n} z^{-n} .
\end{aligned}
$$

It is easy to see that $\partial_{z} \overline{\beta(z)}=\beta(z)$ and $\partial_{z} \overline{\beta(z)_{+}}=\beta(z)_{+}$. For

$$
A, B \in\{\alpha(z), \overline{\beta(z)} \mid \alpha, \beta \in H\}
$$

we define $\langle A, B\rangle=\left[A, B_{+}\right]$. Then it is easy to show (see [Frenkel et al. 1988]) that $\langle\overline{\alpha(z)}, \overline{\beta(w)}\rangle=(\alpha, \beta) \log (z-w)$ for $\alpha, \beta \in H$, which then implies

$$
\begin{aligned}
& \langle\alpha(z), \overline{\beta(w)}\rangle=(\alpha, \beta)(z-w)^{-1}, \\
& \langle\overline{\alpha(z)}, \beta(w)\rangle=-(\alpha, \beta)(z-w)^{-1}, \\
& \langle\alpha(z), \beta(w)\rangle=(\alpha, \beta)(z-w)^{-2},
\end{aligned}
$$

where the formal power series in $z$ and $w$ are understood to be expanded in the second variable $w$.

Define the usual normal ordering : : as in [Frenkel et al. 1988]. Then we have for $\alpha \in H$

$$
: \alpha(z) \beta(w):=\alpha(z) \beta(w)-\langle\alpha(z), \beta(w)\rangle,
$$

and, for $\alpha \in \Gamma_{0}$,

$$
: e^{\overline{\alpha(z)}}:=e^{\alpha} z^{\alpha(0)} \exp \left(-\sum_{n<0} \frac{\alpha(n)}{n} z^{-n}\right) \exp \left(-\sum_{n>0} \frac{\alpha(n)}{n} z^{-n}\right) .
$$

It is clear that the vertex operators $: e^{\overline{\alpha(z)}}$, for $\alpha \in \Gamma_{0}$, can be formally expanded as a power series in $z$ for which the coefficients are well defined operators acting on the Fock space $V$.

We will need the following result in the study of the bosonic realizations for the toroidal Lie algebra $T\left(A_{1}\right)$; see [Jing and Lyerly 1999]. 
Lemma 3.1. Let $P_{i}(z), Q_{i}(w)$, for $i=1,2$, be fields such that the contractions $\left\langle P_{i}, Q_{j}\right\rangle$ commute with all fields $P_{i}(z), Q_{i}(w)$. Then

$$
\begin{aligned}
& : e^{P_{1}} P_{2}:: e^{Q_{1}} Q_{2}:=: e^{P_{1}} P_{2} e^{Q_{1}} Q_{2}: e^{\left\langle P_{1}, Q_{1}\right\rangle}+: e^{P_{1}} P_{2} e^{Q_{1}}: e^{\left\langle P_{1}, Q_{1}\right\rangle}\left\langle P_{1}, Q_{2}\right\rangle \\
& \quad+: e^{P_{1}} e^{Q_{1}} Q_{2}: e^{\left\langle P_{1}, Q_{1}\right\rangle}\left\langle P_{2}, Q_{1}\right\rangle+: e^{P_{1}} e^{Q_{1}}: e^{\left\langle P_{1}, Q_{1}\right\rangle}\left(\left\langle P_{2}, Q_{2}\right\rangle+\left\langle P_{1}, Q_{2}\right\rangle\left\langle P_{2}, Q_{1}\right\rangle\right) .
\end{aligned}
$$

For $\alpha, \beta \in \Gamma_{1}$, we have, from [Frenkel et al. 1988], the identity

$$
: e^{\overline{\alpha(z)}}:: e^{\overline{\beta(w)}}:=: e^{\overline{\alpha(z)}} e^{\overline{\beta(w)}}:(z-w)^{(\alpha, \beta)} .
$$

Inductively one can show, for $\beta_{1}, \ldots, \beta_{k} \in \Gamma_{0}$, the following Wick theorem

$$
: e^{\overline{\beta_{1}\left(z_{1}\right)}}: \cdots: e^{\overline{\beta_{k}\left(z_{k}\right)}}:=: e^{\overline{\beta_{1}\left(z_{1}\right)}} \cdots e^{\overline{\beta_{k}\left(z_{k}\right)}}: \prod_{i<j}\left(z_{i}-z_{j}\right)^{\left(\beta_{i}, \beta_{j}\right)} .
$$

Corollary 3.2. For $\alpha, \beta \in \Gamma_{0}$ and $\gamma, \tau \in H$, suppose $(\alpha, \beta)=0$. Then $\left[: e^{\overline{\alpha(z)}} \gamma(z):,: e^{\overline{\beta(w)}} \tau(w):\right]=: e^{\overline{(\alpha+\beta)(z)}} A(z): z^{-1} \delta\left(\frac{w}{z}\right)+B: e^{\overline{(\alpha+\beta)(z)}}: z^{-1} \partial_{w} \delta\left(\frac{w}{z}\right)$, where $A=(\gamma, \beta) \tau-(\alpha, \tau) \gamma-B \beta \in H$ and $B=(\gamma, \tau)-(\alpha, \tau)(\gamma, \beta) \in \mathbb{C}$.

To give our first representation of the toroidal Lie algebra $T\left(A_{1}\right)$ we consider the lattice

$$
\Gamma:=\frac{1}{k_{0}}\left(\mathbb{Z} a_{0} \oplus \mathbb{Z} a_{1} \oplus \mathbb{Z} b \oplus \mathbb{Z} r\right),
$$

with a symmetric bilinear form determined by

$$
(b, b)=-2 k_{0}, \quad(r, r)=2\left(k_{0}+2\right), \quad\left(a_{i}, a_{j}\right)=k_{0} a_{i j} \quad \text { for } i, j=0,1,
$$

the others being zero. Let $\Gamma_{0}=\frac{1}{k_{0}}\left(\mathbb{Z}\left(a_{0}-b\right)+\mathbb{Z}\left(a_{1}+b\right)\right)$, which is clearly an integral sublattice of $\Gamma$. On the corresponding Fock space $V:=\mathbb{C}\left[\Gamma_{0}^{\star}\right] \otimes S\left(\mathscr{H}^{-}\right)$, we define vertex operators

$$
\begin{aligned}
& X_{0}\left( \pm \alpha_{1}, z\right)=\frac{1}{2}: e^{ \pm \frac{1}{k_{0}} \overline{\left(a_{1}+b\right)(z)}}(b(z) \mp r(z)): \\
& X_{0}\left( \pm \alpha_{0}, z\right)=\frac{1}{2}: e^{ \pm \frac{1}{k_{0}} \overline{\left(a_{0}-b\right)(z)}}(b(z) \pm r(z)):,
\end{aligned}
$$

where $\alpha_{0}, \alpha_{1}$ are the simple roots of the affine Lie algebra $A_{1}^{(1)}$.

Theorem 3.3. Let $k_{0}$ be any nonzero complex number. Then on the Fock space $V$ we have a representation for the toroidal Lie algebra $T\left(A_{1}\right)$. The homomorphism is given by $\not \mapsto \mapsto k_{0}, \alpha_{i}(z) \mapsto a_{i}(z), x\left( \pm \alpha_{i}, z\right) \mapsto X_{0}\left( \pm \alpha_{i}, z\right)$, for $i=0,1$.

Proof. We first write the vertex operators in the form

$$
X_{0}\left( \pm \alpha_{i}, z\right)=\frac{1}{2}: e^{ \pm \frac{1}{k_{0}} \overline{\left(a_{i}-\epsilon_{i} b\right)(z)}}\left(b(z) \pm \epsilon_{i} r(z)\right):,
$$


where $\epsilon_{i}=(-1)^{i}$ for $i=0,1$. We will now show that the operators $a_{i}(z)$ and $X_{0}\left( \pm \alpha_{i}, z\right)$ satisfy the relations $\left(\mathrm{R} 0^{\prime}\right)-\left(\mathrm{R} 4^{\prime}\right)$ of the toroidal Lie algebra $T\left(A_{1}\right)$. In fact, $\left(\mathrm{R} 0^{\prime}\right)$ and $\left(\mathrm{R} 1^{\prime}\right)$ are obvious. For $\left(\mathrm{R} 2^{\prime}\right)$ we have

$$
\begin{aligned}
{\left[a_{i}(z), X_{0}\left( \pm \alpha_{j}, w\right)\right] } & =\frac{1}{2}\left[: a_{i}(z):,: e^{ \pm \frac{1}{k_{0}} \overline{\left(a_{j}-\epsilon_{j} b\right)(z)}}\left(b(z) \pm \epsilon_{j} r(z)\right):\right] \\
& =\frac{1}{2}: e^{ \pm \frac{1}{k_{0}} \overline{\left(a_{j}-\epsilon_{j} b\right)(z)}} A(z): z^{-1} \delta\left(\frac{w}{z}\right)
\end{aligned}
$$

where $A=\left(a_{i}, \pm \frac{1}{k_{0}}\left(a_{j}-\epsilon_{j} b\right)\right)\left(b \pm \epsilon_{j} r\right)= \pm a_{i j}\left(b \pm \epsilon_{j} r\right)$. Therefore

$$
\begin{aligned}
{\left[a_{i}(z), X_{0}\left( \pm \alpha_{j}, w\right)\right] } & = \pm \frac{1}{2} a_{i j}: e^{ \pm \frac{1}{k_{0}} \overline{\left.a_{j}-\epsilon_{j} b\right)(z)}}\left(b(z) \pm \epsilon_{j} r(z)\right): z^{-1} \delta\left(\frac{w}{z}\right) \\
& = \pm a_{i j} X_{0}\left( \pm \alpha_{j}, z\right) z^{-1} \delta\left(\frac{w}{z}\right)
\end{aligned}
$$

which is the required relation. To prove relation $\left(\mathrm{R} 3^{\prime}\right)$ we have

$$
\begin{aligned}
& {\left[X_{0}\left(\alpha_{i}, z\right), X_{0}\left(\alpha_{j}, w\right)\right]} \\
& \quad=\frac{1}{4}\left[: e^{\frac{1}{k_{0}} \overline{\left(a_{i}-\epsilon_{i} b\right)(z)}}\left(b(z)+\epsilon_{i} r(z)\right):,: e^{-\frac{1}{k_{0}} \overline{\left(a_{j}-\epsilon_{j} b\right)(w)}}\left(b(w)-\epsilon_{j} r(w)\right):\right] \\
& =\frac{1}{4}\left(: e^{\frac{1}{k_{0}} \overline{\left(a_{i}-a_{j}-\epsilon_{i} b+\epsilon_{j} b\right)(z)}} A(z): z^{-1} \delta\left(\frac{w}{z}\right)+B: e^{\frac{1}{k_{0}} \overline{\left(a_{i}-a_{j}-\epsilon_{i} b+\epsilon_{j} b\right)(z)}}: z^{-1} \partial_{w} \delta\left(\frac{w}{z}\right)\right),
\end{aligned}
$$

where, by applying Corollary 3.2,

$$
\begin{aligned}
B= & \left(b+\epsilon_{i} r, b-\epsilon_{j} r\right)-\left(\frac{a_{i}-\epsilon_{i} b}{k_{0}}, b-\epsilon_{j} r\right)\left(b+\epsilon_{i} r,-\frac{a_{j}-\epsilon_{j} b}{k_{0}}\right) \\
= & -2 k_{0}-2 \epsilon_{i} \epsilon_{j} k_{0}, \\
A= & \left(b+\epsilon_{i} r,-\frac{a_{j}-\epsilon_{j} b}{k_{0}}\right)\left(b-\epsilon_{j} r\right) \\
& \quad-\left(\frac{a_{i}-\epsilon_{i} b}{k_{0}}, b-\epsilon_{j} r\right)\left(b+\epsilon_{i} r\right)-\left(-2 k_{0}-2 \epsilon_{i} \epsilon_{j} k_{0}\right)\left(-\frac{a_{j}-\epsilon_{j} b}{k_{0}}\right) \\
= & -2\left(1+\epsilon_{i} \epsilon_{j}\right) a_{j} .
\end{aligned}
$$

Therefore, we get

$$
\begin{aligned}
{\left[X_{0}\left(\alpha_{i}, z\right), X_{0}\left(-\alpha_{j}, w\right)\right]=} & -\frac{1}{2}\left(1+\epsilon_{i} \epsilon_{j}\right)\left(: e^{\frac{1}{k_{0}} \overline{\left(a_{i}-a_{j}-\epsilon_{i} b+\epsilon_{j} b\right)(z)}} a_{j}(z): z^{-1} \delta\left(\frac{w}{z}\right)\right. \\
& \left.+k_{0}: e^{\frac{1}{k_{0}} \overline{\left(a_{i}-a_{j}-\epsilon_{i} b+\epsilon_{j} b\right)(z)}}: z^{-1} \partial_{w} \delta\left(\frac{w}{z}\right)\right) \\
= & -\delta_{i j}\left(a_{j}(z) z^{-1} \delta\left(\frac{w}{z}\right)+k_{0} z^{-1} \partial_{w} \delta\left(\frac{w}{z}\right)\right),
\end{aligned}
$$

as required. 
$\left(\mathrm{R} 4^{\prime}\right)$ contains two types of relations. We give only the proof for the "positive" case. The "negative" case can be proved similarly.

$$
\begin{aligned}
& {\left[X_{0}\left(\alpha_{i}, z\right), X_{0}\left(\alpha_{j}, w\right)\right]} \\
& =\frac{1}{4}\left[: e^{\frac{1}{k_{0}} \overline{\left(a_{i}-\epsilon_{i} b\right)(z)}}\left(b(z)+\epsilon_{i} r(z)\right):,: e^{\frac{1}{k_{0}} \overline{\left(a_{j}-\epsilon_{j} b\right)(w)}}\left(b(w)+\epsilon_{j} r(w)\right):\right] \\
& =\frac{1}{4}\left(: e^{\frac{1}{k_{0}} \overline{\left(a_{i}+a_{j}-\epsilon_{i} b-\epsilon_{j} b\right)(z)}} A(z): z^{-1} \delta\left(\frac{w}{z}\right)+B: e^{\frac{1}{k_{0}} \overline{\left(a_{i}+a_{j}-\epsilon_{i} b-\epsilon_{j} b\right)(z)}}: z^{-1} \partial_{w} \delta\left(\frac{w}{z}\right)\right),
\end{aligned}
$$

where, by applying Corollary 3.2,

$$
\begin{aligned}
B & =\left(b+\epsilon_{i} r, b+\epsilon_{j} r\right)-\left(\frac{a_{i}-\epsilon_{i} b}{k_{0}}, b+\epsilon_{j} r\right)\left(b+\epsilon_{i} r, \frac{a_{j}-\epsilon_{j} b}{k_{0}}\right)=2 k_{0}\left(\epsilon_{i} \epsilon_{j}-1\right), \\
A= & \left(b+\epsilon_{i} r, \frac{a_{j}-\epsilon_{j} b}{k_{0}}\right)\left(b+\epsilon_{j} r\right) \\
& \quad-\left(\frac{a_{i}-\epsilon_{i} b}{k_{0}}, b+\epsilon_{j} r\right)\left(b+\epsilon_{i} r\right)-2 k_{0}\left(\epsilon_{i} \epsilon_{j}-1\right)\left(\frac{a_{j}-\epsilon_{j} b}{k_{0}}\right) \\
= & 2\left(1-\epsilon_{i} \epsilon_{j}\right) a_{j} .
\end{aligned}
$$

Therefore $\left[X_{0}\left(\alpha_{i}, z\right), X_{0}\left(\alpha_{i}, w\right)\right]=0$ and, for $i \neq j$,

$\left[X_{0}\left(\alpha_{i}, z\right), X_{0}\left(\alpha_{j}, w\right)\right]$

$$
=: e^{\frac{1}{k_{0}} \overline{\left(a_{i}+a_{j}\right)(z)}} a_{j}(z): z^{-1} \delta\left(\frac{w}{z}\right)-k_{0}: e^{\frac{1}{k_{0}} \overline{\left(a_{i}+a_{j}\right)(z)}}: z^{-1} \partial_{w} \delta\left(\frac{w}{z}\right) .
$$

Clearly, for $i \neq j$, the vertex operator $X_{0}\left(\alpha_{i}, z\right)$ commutes with

$$
: e^{\frac{1}{k_{0}} \overline{\left(a_{i}+a_{j}\right)}(z)} \text {. }
$$

Therefore to complete the proof of relation $\left(\mathrm{R} 4^{\prime}\right)$ we only need to show the identity

$$
\left[X_{0}\left(\alpha_{i}, z_{1}\right),\left[X_{0}\left(\alpha_{i}, z_{2}\right),: e^{\frac{1}{k_{0}} \overline{\left(a_{i}+a_{j}\right)}\left(z_{3}\right)} a_{j}\left(z_{3}\right):\right]\right]=0
$$

for $i \neq j$. Indeed,

$$
\begin{aligned}
& {\left[X_{0}\left(\alpha_{i}, z\right),: e^{\frac{1}{k_{0}} \overline{\left(a_{i}+a_{j}\right)}(w)} a_{j}(w):\right]} \\
& \quad=\frac{1}{2}\left[: e^{\frac{1}{k_{0}} \overline{\left(a_{i}-\epsilon_{i} b\right)}(z)}\left(b+\epsilon_{i} r\right)(z):,: e^{\frac{1}{k_{0}} \overline{\left(a_{i}+a_{j}\right)}(w)} a_{j}(w):\right] \\
& \quad=\frac{1}{2}\left(: e^{\frac{1}{k_{0}} \overline{\left(2 a_{i}+a_{j}-\epsilon_{i} b\right)(z)}} A(z): z^{-1} \delta\left(\frac{w}{z}\right)+B: e^{\frac{1}{k_{0}} \overline{\left(2 a_{i}+a_{j}-\epsilon_{i} b\right)(z)}}: z^{-1} \partial_{w} \delta\left(\frac{w}{z}\right)\right),
\end{aligned}
$$

where, by applying Corollary 3.2,

$$
B=\left(b+\epsilon_{i} r, a_{j}\right)-\left(\frac{a_{i}-\epsilon_{i} b}{k_{0}}, a_{j}\right)\left(b+\epsilon_{i} r, \frac{a_{i}+a_{j}}{k_{0}}\right)=0
$$

and

$$
A=\left(b+\epsilon_{i} r, \frac{a_{i}+a_{j}}{k_{0}}\right) a_{j}-\left(\frac{a_{i}-\epsilon_{i} b}{k_{0}}, a_{j}\right)\left(b+\epsilon_{i} r\right)=2\left(b+\epsilon_{i} r\right) ;
$$


that is

$$
\left[X_{0}\left(\alpha_{i}, z\right),: e^{\frac{1}{k_{0}} \overline{\left(a_{i}+a_{j}\right)}(w)} a_{j}(w):\right]=: e^{\frac{1}{k_{0}} \overline{\left(2 a_{i}+a_{j}-\epsilon_{i} b\right)}(z)}\left(b+\epsilon_{i} r\right): z^{-1} \delta\left(\frac{w}{z}\right) .
$$

Therefore (1) is reduced to the identity

$$
\left[X_{0}\left(\alpha_{i}, z\right),: e^{\frac{1}{k_{0}} \overline{\left(2 a_{i}+a_{j}-\epsilon_{i} b\right)}(w)}\left(b+\epsilon_{i} r\right)(w):\right]=0
$$

for $i \neq j$. The left side is equal to

$$
\begin{aligned}
& \frac{1}{2}\left[: e^{\frac{1}{k_{0}} \overline{\left(a_{i}-\epsilon_{i} b\right)}(z)}\left(b+\epsilon_{i} r\right)(z):,: e^{\frac{1}{k_{0}} \overline{\left(2 a_{i}+a_{j}-\epsilon_{i} b\right)}(w)}\left(b+\epsilon_{i} r\right)(w):\right] \\
& \quad=\frac{1}{2}\left(: e^{\frac{1}{k_{0}} \overline{\left(3 a_{i}+a_{j}-2 \epsilon_{i} b\right)(z)}} A(z): z^{-1} \delta\left(\frac{w}{z}\right)+B: e^{\frac{1}{k_{0}} \overline{\left(3 a_{i}+a_{j}-2 \epsilon_{i} b\right)(z)}}: z^{-1} \partial_{w} \delta\left(\frac{w}{z}\right)\right),
\end{aligned}
$$

where, by applying Corollary 3.2,

$$
B=\left(b+\epsilon_{i} r, b+\epsilon_{i} r\right)-\left(\frac{a_{i}-\epsilon_{i} b}{k_{0}}, b+\epsilon_{i} r\right)\left(b+\epsilon_{i} r, \frac{2 a_{i}+a_{j}-\epsilon_{i} b}{k_{0}}\right)=0
$$

and

$$
A=\left(b+\epsilon_{i} r, \frac{2 a_{i}+a_{j}-\epsilon_{i} b}{k_{0}}\right)\left(b+\epsilon_{i} r\right)-\left(\frac{a_{i}-\epsilon_{i} b}{k_{0}}, b+\epsilon_{i} r\right)\left(b+\epsilon_{i} r\right)=0,
$$

giving the desired identity.

From the construction of the representation for the toroidal Lie algebra given in the previous theorem, it is easy to see that the operators $\alpha_{1}(k)+\alpha_{0}(k)$ act on the Fock space $V$ trivially for all positive integers $k$, which in turn implies that the central elements $\psi(\delta(k))$ act as the zero operator for $k>0$. Therefore the representation is not faithful. Indeed, the quotient space $V(0)$ of the Fock space

$$
\mathbb{C}\left[\Gamma_{0}^{*}\right] \otimes S\left(\mathscr{H}^{-}\right)
$$

defines a representation for the double affine Lie algebra $T_{0}\left(A_{1}\right)$, which is isomorphic to the Lie algebra $T\left(A_{1}\right)$ modulo all central elements of degree other then zero (see Section 2).

Corollary 3.4. The vector space $V(0)$ is endowed with a representation of the double affine Lie algebra $T_{0}\left(A_{1}\right)$ with level- $\left(k_{0}, 0\right)$, under the formula given before Theorem 3.3.

We will study this module structure again in the next section.

To give a faithful representation of the toroidal Lie algebra, we consider the rank-six lattice

$$
\Gamma:=\frac{1}{k_{0}}\left(\mathbb{Z} a_{0} \oplus \mathbb{Z} a_{1} \oplus \mathbb{Z} b \oplus \mathbb{Z} c \oplus \mathbb{Z} d\right) \oplus \frac{1}{k_{0}+2} \mathbb{Z} r,
$$


with the symmetric bilinear form determined by $(b, b)=-2 k_{0}, \quad(r, r)=2\left(k_{0}+2\right), \quad(c, d)=k_{0}, \quad\left(a_{i}, a_{j}\right)=k_{0} a_{i j} \quad$ for $i, j=0,1$, all others being zero. Then

$$
\Gamma_{0}:=\frac{1}{k_{0}} \mathbb{Z}\left(a_{0}-b\right)+\frac{1}{k_{0}} \mathbb{Z}\left(a_{1}+b\right)+\frac{1}{k_{0}} \mathbb{Z} c
$$

is clearly an integral sublattice of $\Gamma$. Let $\Gamma_{0}^{\star}$ be the corresponding additive subgroup of $H=\mathbb{C} \otimes_{\mathbb{Z}} \Gamma$, and $V$ the corresponding Fock space.

We also modify the vertex operators from the previous theorem to the form

$$
\begin{aligned}
& X\left( \pm \alpha_{1}, z\right)=\frac{1}{2}: e^{ \pm \frac{1}{k_{0}} \overline{\left(a_{1}+b\right)(z)}}(b(z) \mp r(z)):, \\
& X\left( \pm \alpha_{0}, z\right)=\frac{1}{2}: e^{ \pm \frac{1}{k_{0}} \overline{\left(a_{0}-b+c\right)(z)}}(b(z) \pm r(z)): .
\end{aligned}
$$

Theorem 3.5. The coefficient operators of the vertex operators $a_{i}(z), X\left( \pm \alpha_{i}, z\right)$, for $i=0,1$, acting on the Fock space $V$, generate a Lie algebra $\mathscr{L}\left(A_{1}\right)$ isomorphic to the toroidal Lie algebra $T\left(A_{1}\right)$, the isomorphism begin given by the linear map $\phi$ defined by

$$
\begin{aligned}
\not & \mapsto k_{0}, \\
\alpha_{1}(z) & \mapsto a_{1}(z), \\
\alpha_{0}(z) & \mapsto a_{0}(z)+c(z), \\
x\left( \pm \alpha_{i}, z\right) & \mapsto X\left( \pm \alpha_{i}, z\right) \quad \text { for } i=0,1 .
\end{aligned}
$$

Therefore, on the Fock space $V$, we have a faithful representation of the toroidal Lie algebra $T\left(A_{1}\right)$.

Proof. We first need to show that the surjective mapping $\phi$ defines a Lie algebra homomorphism from $T\left(A_{1}\right)$ to $\mathscr{L}\left(A_{1}\right)$. It suffices to show that the vertex operators $a_{i}(z), X\left( \pm \alpha_{i}, z\right)$ satisfy the corresponding power series identities $\left(\mathrm{R} 0^{\prime}\right)-\left(\mathrm{R} 4^{\prime}\right)$. The argument is just as in the proof of Theorem 3.3, and we omit it for brevity's sake.

We next use Proposition 2.2 to show that the mapping $\phi$ is indeed an injective homomorphism. For $\alpha=\mu_{1} a_{0}+\mu_{2} a_{1}+\mu_{3} b+\mu_{4} c \in \Gamma_{0}^{\star}$ with $\mu_{i} \in \frac{1}{k_{0}} \mathbb{Z}$, let

$$
e^{\alpha} \otimes \lambda_{1}\left(-n_{1}\right) \cdots \lambda_{k}\left(-n_{k}\right) \in V
$$

We define a $\mathbb{Z} \times Q$-gradation on the Fock space $V$ by setting

$$
\operatorname{deg}\left(e^{\alpha} \otimes \lambda_{1}\left(-n_{1}\right) \cdots \lambda_{k}\left(-n_{k}\right)\right)=\left(n_{1}+\cdots+n_{k}, k_{0} \mu_{1} \alpha_{0}+k_{0} \mu_{2} \alpha_{1}\right) .
$$

With this gradation, the operator $a(n)$, for $a \in H$, is a homogeneous operator of degree $(-n, 0)$. Moreover, if the vertex operator $X\left( \pm \alpha_{i}, z\right)$ is formally expanded 
into power series as

$$
X\left( \pm \alpha_{i}, z\right)=\sum_{m \in \mathbb{Z}} X_{m}\left( \pm \alpha_{i}\right) z^{-m-1}
$$

the coefficient operator $X_{m}\left( \pm \alpha_{i}\right)$ is a homogeneous operator of degree $\left(-m, \pm \alpha_{i}\right)$. Thus the map $\phi$ is a $(\mathbb{Z} \times Q)$-graded Lie algebra homomorphism. To finish the proof of this theorem, we need only show that $\phi$ satisfies the three conditions of Proposition 2.2.

Recall the notation $x_{m}\left( \pm \alpha_{1}+k \delta\right)=\psi^{-1}\left( \pm s^{m} t^{k} \otimes x_{ \pm}\right)$, where $\delta=\alpha_{0}+\alpha_{1}$ is the null root in $Q$. Let

$$
x(\alpha, z)=\sum_{m \in \mathbb{Z}} x_{m}(\alpha) z^{-m-1} \quad \text { for } \alpha= \pm \alpha_{1}+k \delta .
$$

Then it is easy to show that $\phi: x(\alpha, z) \mapsto X(\alpha, z)$, where $\alpha= \pm \alpha_{1}+k \delta$, and

$$
X\left( \pm \alpha_{1}+k \delta, z\right)=\frac{1}{2}: e^{ \pm \frac{1}{k_{0}} \overline{\left(a_{1}+b+k\left(a_{0}+a_{1}\right)+k c\right)(z)}}(b(z) \mp r(z)): .
$$

Applying Corollary 3.2 again we have

$$
\begin{aligned}
{\left[X\left(\alpha_{1}+k \delta, z\right), X\left(-\alpha_{1}\right.\right.} & -k \delta, w)] \\
& =-k_{0} z^{-1} \partial_{w} \delta\left(\frac{w}{z}\right)-\left(a_{1}+k\left(a_{0}+a_{1}\right)+k c\right)(z) z^{-1} \delta\left(\frac{w}{z}\right) .
\end{aligned}
$$

This gives

$$
\left[X_{m}\left(\alpha_{1}+k \delta\right), X_{-m}\left(-\alpha_{1}-k \delta\right)\right]=-a_{1}(0)-k\left(a_{0}+a_{1}\right)(0)-k c(0)-m k_{0},
$$

which is clearly a nonzero operator for any $m, k \in \mathbb{Z}$. Thus $\phi$ is injective on the onedimensional subspace $T_{m}^{\alpha}=\mathbb{C} x_{m}(\alpha)$ for any real root $\alpha= \pm \alpha_{1}+k \delta$ and $k, m \in \mathbb{Z}$. Moreover,

$$
\phi(\delta(k))=a_{0}(k)+a_{1}(k)+c(k)
$$

is also a nonzero operator, and $\phi$ is clearly injective on $\mathbb{C} \delta(0)+\mathbb{C} \phi$.

Finally, we need to show that, for $m, k \neq 0$,

$$
\begin{array}{r}
{\left[X_{m}\left(\alpha_{1}+k \delta\right), X_{0}\left(-\alpha_{1}\right)\right]-\left[X_{0}\left(\alpha_{1}+k \delta\right), X_{m}\left(-\alpha_{1}\right)\right] \neq 0,} \\
{\left[X_{1}\left(\alpha_{1}+k \delta\right), X_{-1}\left(-\alpha_{1}\right)\right]-\left[X_{-1}\left(\alpha_{1}+k \delta\right), X_{1}\left(-\alpha_{1}\right)\right] \neq 0 .}
\end{array}
$$

By Corollary 3.2,

$$
\begin{gathered}
{\left[X\left(\alpha_{1}+k \delta, z\right), X\left(-\alpha_{1}, w\right)\right]+\left[X\left(-\alpha_{1}, z\right), X\left(\alpha_{1}+k \delta, w\right)\right]} \\
=-2 k_{0}: e^{\frac{1}{k_{0}} \overline{\left(k a_{0}+k a_{1}+k c\right)(z)}}: z^{-1} \partial_{w} \delta\left(\frac{w}{z}\right) \\
+k: e^{\frac{1}{k_{0} \overline{\left(k a_{0}+k a_{1}+k c\right)(z)}}}\left(a_{0}(z)+a_{1}(z)+c(z)\right): z^{-1} \delta\left(\frac{w}{z}\right)
\end{gathered}
$$


which gives

$$
\begin{aligned}
& {\left[X\left(\alpha_{1}+k \delta, z\right), X_{0}\left(-\alpha_{1}\right)\right]-\left[X_{0}\left(\alpha_{1}+k \delta\right), X\left(-\alpha_{1}, z\right)\right]} \\
& =k: e^{\frac{1}{k 0} \overline{\left(k a_{0}+k a_{1}+k c\right)(z)}}\left(a_{0}(z)+a_{1}(z)+c(z)\right): .
\end{aligned}
$$

To see that the coefficient of $z^{-m-1}$ in the expression on the right is nonzero for $m \neq 0$, we notice that

$$
\begin{aligned}
{\left[: e^{\frac{1}{k_{0}} \overline{\left(k a_{0}+k a_{1}+k c\right)(z)}}\left(a_{0}(z)+a_{1}(z)+c(z)\right):,: e^{-\frac{1}{k_{0}} \overline{\left(k a_{0}+k a_{1}+k c\right)(z)}} d(z):\right] } \\
\quad=k_{0} z^{-1} \partial_{w} \delta\left(\frac{w}{z}\right) .
\end{aligned}
$$

The coefficient of $z^{-m-1}$ on the right-hand side of the previous identity is $k_{0} m w^{m-1}$, which is nonzero whenever $m \neq 0$. This proves the first line in (2), while the second can be proved by a similar argument which is omitted here. Therefore $\phi$ is an isomorphism of Lie algebras.

Corollary 3.6. For any fixed $k_{1} \in \mathbb{Z}$, define

$$
V\left(k_{1}\right)=e^{k_{1} d+\Gamma_{0}} \otimes S\left(\mathscr{H}^{-}\right) .
$$

Then the vector space $V\left(k_{1}\right)$ is endowed with a representation of the toroidal Lie algebra $T\left(A_{1}\right)$ with level- $\left(k_{0}, k_{1}\right)$.

\section{Module structure}

We now define a smaller module from our Fock space representation via the socalled screening operator. We will only consider the case when $c=0$.

For given $j_{0}, j_{1}, l_{1}, l_{2} \in \mathbb{C}$ with $j_{0}+j_{1} \in \mathbb{Z} \frac{k_{0}}{2}$, set

$$
v_{j_{0}, j_{1}, l_{1}, l_{2}}:=e^{j_{0} \frac{a_{0}}{k_{0}}} e^{j_{1} \frac{a_{1}}{k_{0}}} e^{l_{1} \frac{b}{k_{0}}} e^{-l_{2} \frac{r}{k_{0}+2}} .
$$

We define the Fock space $F_{j_{0}, j_{1}, l_{1}, l_{2}}$ to be the space $S\left(\mathscr{H}^{-}\right) v_{j_{0}, j_{1}, l_{1}, l_{2}}$. Then the vertex operators $X\left( \pm \alpha_{i}, z\right)$ are well defined on $F_{j_{0}, j_{1}, l_{1}, l_{2}}$, provided that $2\left(j_{1}-l_{1}\right)$ and $2\left(j_{0}+l_{1}\right)$ are integers. It is clear that the vertex operators satisfy

$$
\begin{aligned}
& X\left( \pm \alpha_{0}, z\right): F_{j_{0}, j_{1}, l_{1}, l_{2}} \longrightarrow F_{j_{0} \pm 1, j_{1}, l_{1} \mp 1, l_{2}}, \\
& X\left( \pm \alpha_{1}, z\right): F_{j_{0}, j_{1}, l_{1}, l_{2}} \longrightarrow F_{j_{0}, j_{1} \pm 1, l_{1} \pm 1, l_{2}} .
\end{aligned}
$$

Introduce a screening operator $S_{0}: F_{j_{0}, j_{1}, l_{1}, l_{2}} \rightarrow F_{j_{0}, j_{1}, l_{1}+\frac{k_{0}}{2}, l_{2}+\frac{k_{0}+2}{2}}$ by setting

$$
S(z)=: e^{\frac{1}{2} \overline{(b(z)-r(z))}}:=\sum_{n} S_{n} z^{-n-1} .
$$

This is well defined provided that $l_{1}-l_{2} \in \mathbb{Z}$. 


\section{Proposition 4.1.}

$$
\begin{aligned}
\left\{X\left(\alpha_{1}, z\right), S(w)\right\} & =\frac{\partial}{\partial w}\left(: e^{\frac{1}{k_{0}},\left(a_{1}+b\right)(w)+\frac{1}{2}(b-r)(w)}: \frac{1}{z-w}\right), \\
\left\{X\left(-\alpha_{1}, z\right), S(w)\right\} & =0 \\
\left\{X\left(-\alpha_{0}, z\right), S(w)\right\} & =0 \\
\left\{X\left(\alpha_{0}, z\right), S(w)\right\} & =\frac{\partial}{\partial w}\left(: e^{\frac{1}{k_{0}\left(a_{0}+b\right)(w)+\frac{1}{2}(b-r)(w)}}: \frac{1}{z-w}\right) .
\end{aligned}
$$

Proof. Let

$$
\begin{aligned}
& \phi\left(\alpha_{1}, z\right)=\phi\left(-\alpha_{0}, z\right):=\frac{1}{2}: e^{\overline{\frac{1}{k_{0}} b(z)}}(b(z)-r(z)):, \\
& \phi\left(\alpha_{0}, z\right)=\phi\left(-\alpha_{1}, z\right):=\frac{1}{2}: e^{\overline{-\frac{1}{k_{0}} b(z)}}(b(z)+r(z)):
\end{aligned}
$$

be the parafermions. It follows from Lemma 3.1 that $\phi\left(\alpha_{1}, z\right) S(w)$

$$
\begin{aligned}
& \sim \frac{1}{2}: e^{\overline{\frac{1}{k_{0}} b(z)}}(b-r)(z) e^{\overline{\frac{1}{2}(b-r)(w)}}: \frac{1}{z-w}+\frac{1}{2}: e^{\overline{\frac{1}{k_{0}} b(z)+\frac{1}{2}(b-r)(w)}}: \frac{2}{(z-w)^{2}} \\
& \sim \frac{\partial}{\partial w}\left(: e^{\overline{\frac{1}{k_{0}} b(w)+\frac{1}{2}(b-r)(w)}}: \frac{1}{z-w}\right) .
\end{aligned}
$$

Let $d$ be the zero mode of $S(z): d=\int S(z) d z$. It is easy to check that the anticommutator $\{S(z), S(z)\}=0$, thus $d$ gives rise to a complex of vector spaces:

$$
\begin{aligned}
& \cdots \longrightarrow F_{j_{0}, j_{1}, l_{1}-\frac{k_{0}}{2}, l_{2}-\frac{k_{0}+2}{2}} \longrightarrow \\
& F_{j_{0}, j_{1}, l_{1}, l_{2}} \longrightarrow \\
& \quad F_{j_{0}, j_{1}, l_{1}+\frac{k_{0}}{2}, l_{2}+\frac{k_{0}+2}{2}} \longrightarrow F_{j_{0}, j_{1}, l_{1}+k_{0}, l_{2}+k_{0}+2} \longrightarrow \cdots
\end{aligned}
$$

We can define the restricted $T(A)$-submodule using Proposition 4.1. Given $l$ we define a $T(A)$-submodule

$$
F_{l}=\bigoplus_{j_{1} \in l+\mathbb{Z}, j_{0} \in-l+\mathbb{Z}} \operatorname{ker}\left(d: F_{j_{0}, j_{1}, j_{1}, l} \rightarrow F_{\left.j_{0}, j_{1}, j_{1}+\frac{k_{0}}{2}, l+\frac{k_{0}+2}{2}\right)} .\right.
$$

Theorem 4.2. The operator $d$ commutes or anticommutes with elements of the toroidal algebra $T\left(A_{1}\right)$ and $d^{2}=0$. Moreover we have the long exact sequence

$$
\begin{aligned}
0 \longrightarrow F_{l} \longrightarrow \bigoplus_{j_{0}, j_{1}} F_{j_{0}, j_{1}, j_{1}, l} \longrightarrow \bigoplus_{j_{0}, j_{1}} F_{j_{0}, j_{1}, j_{1}+\frac{k_{0}}{2}, l+\frac{k_{0}+2}{2}} & \\
& \longrightarrow \bigoplus_{j_{0}, j_{1}} F_{j_{0}, j_{1}, j_{1}+k_{0}, l+k_{0}+2} \longrightarrow \cdots
\end{aligned}
$$

where the maps from $\bigoplus_{j_{0}, j_{1}} F_{j_{0}, j_{1}, j_{1}, l}$ onward are $\bigoplus d$ and the summations run through $j_{0} \in-l+\mathbb{Z}$ and $j_{1} \in l+\mathbb{Z}$. 
Proof. We introduce the operator $S^{\star}(z)=e^{\overline{-\frac{1}{2}(b(z)-r(z))}}=\sum_{n} S_{n}^{*} z^{-n}$, and set $d^{\star}=$ $S_{0}^{\star}$. It is easy to see that $\left\{S(z), S^{\star}(w)\right\}=1$. Hence $d d^{\star}+d^{\star} d=1$, and we already knew that $d^{2}=0$. Thus the following long sequence of vector spaces is exact:

$$
0 \rightarrow \operatorname{ker}_{F_{j_{0}, j_{1}, j_{1}, l}} d \rightarrow F_{j_{0}, j_{1}, j_{1}, l} \rightarrow F_{j_{0}, j_{1}, j_{1}+\frac{k_{0}}{2}, l+\frac{k_{0}+2}{2}} \rightarrow F_{j_{0}, j_{1}, j_{1}+k_{0}, l+k_{0}+2} \rightarrow \cdots
$$

Taking the direct sum we obtain Theorem 4.2.

Since $a_{0}(n)+a_{1}(n)$ acts trivially we can modulo the relation and define

$$
\tilde{F}_{l}=F_{l} /\left(a_{0}(n)+a_{1}(n) ;-n \in \mathbb{N}\right) ;
$$

then it is also a $T\left(A_{1}\right)$-module and the results in Proposition 4.1 obviously hold for the module $\tilde{F}_{l}$. If we further modulo $a_{1}(0)+a_{0}(0)$ we will obtain the Verma module for the affine Lie algebra generically.

Using the exact sequence we can compute the character for the module $\tilde{F}_{l}$ as follows.

Theorem 4.3. The character of the $T\left(A_{1}\right)$-module $\tilde{F}_{l}$ is given by

$$
\operatorname{ch}\left(\tilde{F}_{l}\right)=\sum_{s=0}^{\infty}(-1)^{s} \frac{\sum_{\alpha \in \bar{Q}} e^{-l \frac{r}{k_{0}+2}+s\left(\frac{k_{0}+2}{2} r+\frac{k_{0}}{2} b\right)} e^{\alpha}}{\prod\left(e^{-\delta_{1}}\right) \prod\left(e^{-\delta_{b}}\right) \prod\left(e^{-\delta_{r}}\right)}
$$

where

$$
\prod(x)=\prod_{m>0}\left(1-x^{m}\right) \quad \text { and } \quad \bar{Q}=\frac{1}{k_{0}}\left(\mathbb{Z} \alpha_{1}+\mathbb{Z} b\right) .
$$

\section{References}

[Berman and Billig 1999] S. Berman and Y. Billig, "Irreducible representations for toroidal Lie algebras”, J. Algebra 221:1 (1999), 188-231. MR 2000k:17004 Zbl 0942.17016

[Eswara Rao and Moody 1994] S. Eswara Rao and R. V. Moody, "Vertex representations for $n$ toroidal Lie algebras and a generalization of the Virasoro algebra", Comm. Math. Phys. 159:2 (1994), 239-264. MR 94m:17028 Zbl 0808.17018

[Fabbri and Moody 1994] M. A. Fabbri and R. V. Moody, "Irreducible representations of Virasorotoroidal Lie algebras", Comm. Math. Phys. 159:1 (1994), 1-13. MR 95d:17031 Zbl 0796.17024

[Fer̆gin and Frenkel' 1988] B. L. Fer̆gin and È. V. Frenkel', "A family of representations of affine Lie algebras", Uspekhi Mat. Nauk 43:5 (1988), 227-228. In Russian; translated in Russian Math. Surveys 43:5 (1988), 221-222. MR 89k:17016 Zbl 0657.17013

[Frenkel et al. 1988] I. Frenkel, J. Lepowsky, and A. Meurman, Vertex operator algebras and the Monster, Pure and Applied Mathematics 134, Academic Press, Boston, 1988. MR MR996026 (90h:17026) Zbl 0674.17001

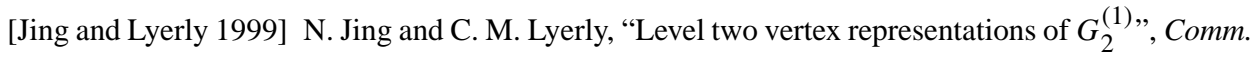
Algebra 27:9 (1999), 4355-4362. MR 2000g:17035 Zbl 1007.17019

[Larsson 1999] T. A. Larsson, "Lowest-energy representations of non-centrally extended diffeomorphism algebras", Comm. Math. Phys. 201:2 (1999), 461-470. MR 2000c:17042 Zbl 0936.17025 
[Moody et al. 1990] R. V. Moody, S. E. Rao, and T. Yokonuma, "Toroidal Lie algebras and vertex representations”, Geom. Dedicata 35:1-3 (1990), 283-307. MR 91i:17032 Zbl 0704.17011

[Nemeschansky 1989] D. Nemeschansky, "Fergin-Fuchs representation of $\widehat{s u}(2)_{k}$ Kac-Moody algebra”, Phys. Lett. B 224:1-2 (1989), 121-124. MR 90g:17026

[Tan 1999] S. Tan, "Principal construction of the toroidal Lie algebra of type $A_{1}$ ", Math. Z. 230:4 (1999), 621-657. MR 2001d:17030 Zbl 0932.17028

[Wakimoto 1986] M. Wakimoto, "Fock representations of the affine Lie algebra $A_{1}^{(1), ", C o m m . ~ M a t h . ~}$ Phys. 104:4 (1986), 605-609. MR 87m:17011 Zbl 0587.17009

Received October 2, 2002. Revised March 18, 2004.

\author{
NAIHUAN JING \\ DEPARTMENT OF MATHEMATICS \\ North CAROLINA STATE UNIVERSITY \\ RALEIGH, NC 27695 \\ UNITED STATES \\ FACULTY OF MATHEMATICS \\ HUBEI UNIVERSITY \\ WUHAN, HUBEI 430064 \\ CHINA \\ jing@math.ncsu.edu \\ KAILASH MisRA \\ DEPARTMENT OF MATHEMATICS \\ North Carolina State University \\ RALEIGH, NC 27695 \\ UNITED STATES \\ misra@math.ncsu.edu \\ SHAOBIN TAN \\ DEPARTMENT OF MATHEMATICS \\ XiAMEN UNIVERSITY \\ XiAMEN, FUJIAN 361005 \\ CHINA \\ tans@jingxian.xmu.edu.cn
}

